\title{
Stroke Caregiver Education: From the Caregivers' Perspectives
}

\author{
Kaitlin M Ryczek, Amanda M Conway, Joanna W Podosek and Lisa Jean Knecht-Sabres* \\ Midwestern University, Occupational Therapy Program, USA
}

Submission: November 24, 2021; Published: December 20, 2021

*Corresponding author: Lisa Knecht-Sabres, Midwestern University, Occupational Therapy Program, Downers Grove, IL, USA

Abstract

Background: Caregivers of stroke survivors report that the education received from providers is unsuitable to their needs. Few studies have investigated methods that are beneficial in promoting caregivers' comfort in their new role. Even fewer studies focus on education provided by occupational therapy (OT) practitioners.

Objectives: This study explored caregivers' perceptions of the education provided by OT practitioners.

Methods: Researchers utilized a convergent parallel mixed methods design. Participants $(n=5)$ completed a survey to gather data regarding education provision. Individual interviews were conducted to better understand the lived experience. Rigor was enhanced through the utilization of audit trails, constant comparison, multiple coders, expert examination, and triangulation.

Results: $60 \%$ of participants felt anxious regarding their abilities; $40 \%$ of participants felt confident and prepared to assist with their loved one's daily needs; $20 \%$ felt unprepared to address challenges at home; and $40 \%$ were neutral about OT education being tailored to their needs. Three themes emerged from the qualitative data: strengths of education provision, shortfalls, and future recommendations.

Conclusion: OTs are not fully addressing caregivers' needs to prepare them for their new role. OTs should consider incorporating more opportunities for collaboration and training with caregivers, ensure that they are meeting the caregivers' preferences in relation to delivery, time, and content of education, as well as make sure that they are including psychosocial resources and occupation-based and client relevant activities to help facilitate their loved one's recovery.

Keywords: Stroke; Occupational therapy; Caregivers; Education; Confidence

\section{Stroke Caregiver Education: From the Caregivers' Perspective}

Strokes are one of the most rapidly occurring health events presently. Approximately one-third of individuals who experience a stroke face subsequent disability from the event [1]. Such impairments can have a significant impact on one's ability to perform activities of daily living [2]. Consequently, loved ones are often forced to take on a new, unplanned role as primary caregiver.

Caregiving typically requires the acquisition of new skills, such as assisting with basic activities of daily living (ADLs), helping with functional mobility, and managing difficult emotions/behaviors. This process can be arduous and overwhelming for individuals with no prior experience or intention to take on such a role. Providing caregivers with information that satisfies their needs and thoroughly prepares them to carry out duties is not only key to the stroke survivors' quality of life, but critical to the caregivers' self-efficacy in their new role in the home environment as well [3]. As experts in fostering participation in meaningful activities and roles, occupational therapists (OTs) are significant contributors in the educational process; however, numerous studies continuously demonstrate how therapists fall short in providing education that promotes the individuals' sense of confidence and competence [4-7]. Even though the basic foundation of educational topics for caregivers have been identified and prioritized, including how to provide assistance for their loved ones, few studies have explored the caregivers' perceptions of the educational methods that are most beneficial and to what extent education provision by OTs promotes confidence and preparedness of caregivers in their new role.

\section{Literature Review}

Caregivers cited the education process as well as the information supplied by providers, as ill-suited to their needs and preferences. More specifically, dissatisfaction has centered around topics such as delivery, educational content, timing, and the quality of communication between providers and caregivers [4-7]. Consideration of the caregivers' perceptions within each of these contexts is imperative as they all play an important role in promoting individuals' confidence and comfort in the provision of care. 


\section{Delivery of information}

Caregivers reported experiences of not knowing who to go to for specific informational needs [4], poor attitudes from professionals during the recovery phase [3], and an overall perception of being a nuisance to providers who appear, "too rushed, stressed, or lazy" [6]. While a portion of caregivers expressed satisfaction with obtaining adequate education from health providers, most emphasized this outcome would likely not have occurred had they not actively sought professionals out with questions [6,8]. In fact, Creasy et al. [8] stated caregivers characterized as less assertive during the educational process are typically less prepared upon discharge.

Caregivers also described the exchange of information as predominantly one-sided and that health providers readily offered large amounts of information without genuinely taking the time to listen to the input of those who will be playing a significant role in the stroke victim's life, health, and wellbeing [9]. According to caregivers, this limits the potential to customize education towards their specific needs. Ultimately, this is a detriment to the educational experience, as caregivers generally exude more assurance about assuming their new role when providers encourage active collaboration during interactions [8].

\section{Timing}

Prior to discharge from rehabilitation, it is common practice for practitioners to provide caregiver education all at once with the assumption that the careers are able to demonstrate what they have learned [10]. Additionally, these family encounters typically occur shortly before discharge and at a time when caregivers are given an abundance of information all at once [11]. Within this process, caregivers find themselves in a predicament of information overload and at a time when anxiety levels high [12]. Thus, caregivers are faced with new challenges regarding the return to home and are presented with an expansive list on how to care for their loved ones on their own. This bombardment of information from the healthcare team is often very overwhelming and is not retained [2]. In fact, several researchers found that caregivers often have difficulty recalling information postdischarge and upon return to the home environment $[9,13]$. Thus, there is a consensus that it would be more beneficial to expand educational opportunities and continue them throughout the rehabilitation process. For example, stroke patients have found a weekend visit home prior to discharge to be beneficial in identifying problems regarding the transition. These experiences provide an opportunity for caregivers to ask the rehabilitation team questions, collaborate, and problem-solve to develop contextual strategies in order to manage the residual deficits of their loved ones [3]. Moreover, caregivers have also reported that they would like to remain active and participate in sessions to receive further education throughout the care continuum
[12]. Such services and resources are beneficial in supporting caregivers in their transition home and enhancing their selfefficacy in their abilities to navigate new challenges and obstacles that arise following discharge [14].

\section{Educational content}

Studies have found caregivers were not prepared for the emotional aspects of caregiving such as managing the stroke survivor's emotions and behavior, as well as their own stress $[7,15]$. Due to high rates of depression in caregivers and individuals post-stroke, emotional management (via coping strategies, antidepressants, or support groups) is important for caregiver's quality of life. Many caregivers expressed concern for their mental health and the emotional management of their loved one [8]. Yet, there is minimal education regarding emotional management provided to individuals before discharge $[9,15]$. In fact, in a study by Roy [6], multiple participants reported not receiving any information on stroke support groups and other resources available to them. It is important to provide education that is individualized and addresses each caregiver's specific information needs and concerns [16,13]. Overall, when it comes to meeting and satisfying caregivers' preferences across all areas of education, the literature suggests there is room for significant improvement.

While numerous qualitative studies have explored discrepancies associated with delivery of education, content, timing, and the extent of quality communication between providers and caregivers [4-7], few, if any, have investigated the methods that are particularly beneficial in promoting individuals' confidence and comfort in their new role as caregiver after discharge. Likewise, even fewer studies have focused on education provided by OTs. Thus, the purpose of this study was to explore (1) what particular methods employed by OTs promoted confidence and comfort in an individuals' caretaking abilities and (2) what methods caregivers believe would have better promoted their preparedness in the transition to the home environment.

\section{Methods}

\section{Design}

A convergent parallel mixed methods design was chosen for this study [15]. The collection and analysis of both quantitative and qualitative allowed the researchers to draw on the strengths of both methodologies and obtain a more comprehensive and accurate finding [17]. More specifically, collecting both sets of data permitted the researchers to not only explore the caregivers' general perceptions of education provided by OTs, but also obtain a more complete understanding of how particular methods employed during the educational process influenced the amount of comfort and confidence in their caretaking abilities. This study was approved by the University's Institutional Review Board. 


\section{Data collection}

Researchers gathered both quantitative and qualitative data concurrently during the data collection phase. A researcherdeveloped survey was used to gather quantitative data (Appendix A). This survey was informed by the literature and modified according to expert opinion. Qualitative data was gathered through the administration of a semi-structured interview to better understand the lived experiences of caregivers with stroke education. More specifically, the interviews collected information regarding how confident caregivers felt in their caretaking abilities after receiving education, what their educational experience was like with the OT, what methods of education they found beneficial in fostering comfort and confidence in their abilities, and what methods they believed should be incorporated into education in the future. Questions included in the Interview Guide (Appendix B) were built from other studies and gaps found in the literature. Interviews were audiotaped and transcribed verbatim.

\section{Measures}

The researcher-developed survey included content related to nature of education received, the educational method(s) used by the OT, the frequency of educational training sessions, the timing of the education, perceptions related to their experience, as well as basic demographic information. Items on the survey included forced-choice questions, open-ended questions, as well as items on a Likert scale. Questions on a Likert scale were designed to gain information regarding the caregivers' perspectives of their overall educational experience in addition to their self-perception about their caretaking abilities in the home environment, and were coded as "strongly disagree", "disagree", "neutral", "agree", and "strongly agree".

\section{Procedures}

Recruitment occurred from June 2019 to September 2019. The researchers used purposive and snowball sampling to recruit informal caregivers of stroke survivors. Researchers contacted the directors of several different stroke support groups in the Chicagoland area for permission to attend a monthly meeting to pass out flyers regarding this research opportunity. Flyers were also posted in a variety of community centers to announce the study. Inclusion criteria for informal caregivers included: (1) ability to speak English in order to provide consent and participate in an interview; (2) being a caregiver of someone who experienced a stroke in the past ten years; (3) an individual who is not compensated for caring services; (4) a caregiver who received caretaking education from a licensed OT practitioner in an inpatient setting; and (5) age of eighteen years or older. Individuals who did not meet the above criteria were excluded.

Individuals interested in participating in the study were offered an opportunity to participate in a focus group with other caregivers of stroke survivors. The option to complete interviews over the phone was made available if the participant was unable to attend the focus group due to transportation issues or scheduling conflicts. All participants opted to provide qualitative data via a phone interview versus attending the focus group. All participants completed the informed consent process prior to data collection.

Prior to the interview, participants were asked to complete the researcher-developed survey. Then, researchers conducted a semistructured interview to collect qualitative data. Each interview lasted approximately thirty to sixty minutes. Participants were not compensated for their participation in the study.

\section{Data analysis}

Quantitative data from the survey was entered into a Microsoft Excel spreadsheet and was analyzed using descriptive statistics. Researchers searched for themes and analyzed information from the qualitative data using inductive thematic analysis $[18,17]$. Thematic analysis was used to identify patterns and similar themes across participants and allowed meaning to emerge from the raw data [18]. With this approach, each researcher read transcripts and coded independently; researchers then came together to collaborate and identify consistent themes until consensus was reached. Quantitative and qualitative results were merged to identify patterns across data sets. Peer debriefing and expert examination was used throughout the data analysis process. As a result, trustworthiness of data was enhanced through constant comparison, multiple coders, expert examination, and triangulation.

\section{Results}

\section{Participants}

A total of 5 individuals across 3 different states participated in the study $(n=5)$. All participants were at least 50 years of age. The participants had varying levels of education, yet the majority of participants were retired and had a graduate degree. The time since their loved one had a stroke ranged from 1 to 8 years, with a mean of 4.2 years. Please see (Table 1) for more specific demographic data.

\section{Quantitative data}

Quantitative data from the survey revealed that the majority $(60 \%)$ of the participants reported feeling anxious about their abilities once in the home environment (Figure1). Additionally, only $40 \%$ of the participants felt confident in their abilities to care for their loved one after discharge (Figure 2) and prepared to assist with their loved one's daily needs (Figure 3). Moreover, $20 \%$ strongly agreed that they felt unprepared to address challenges that arose once home (Figure 4) Furthermore, $40 \%$ of the participants indicated that they were neutral about whether the education provided by OTs was tailored to their needs (Figure 5). 


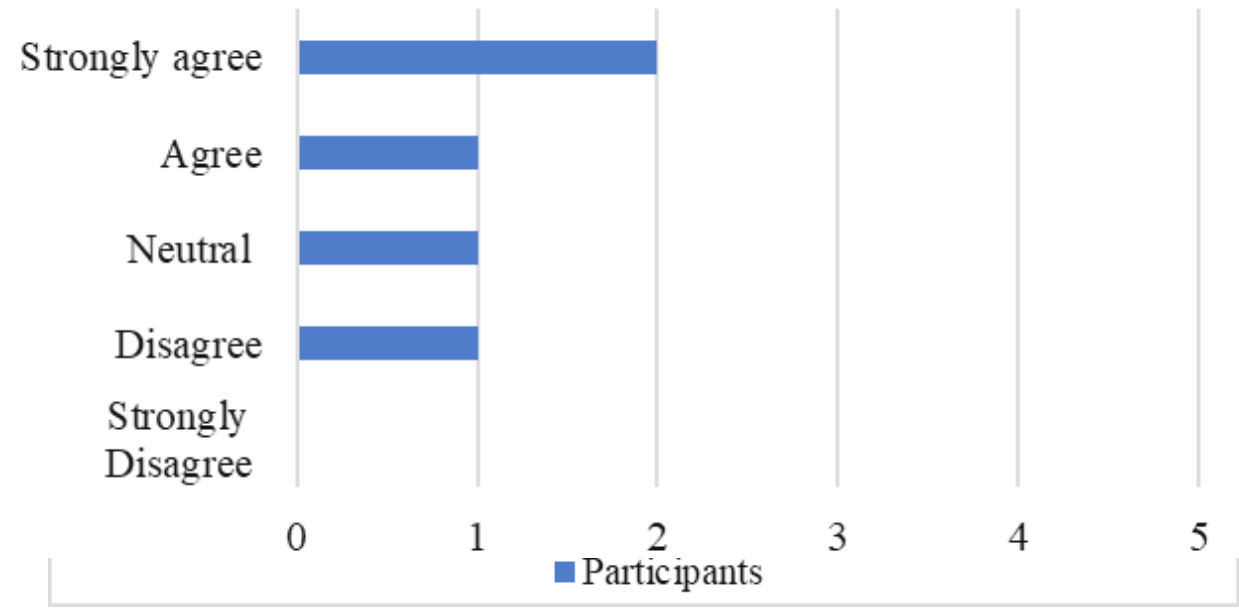

Figure 1: Number of participants indicating "I felt anxious in regard to my caretaking abilities."

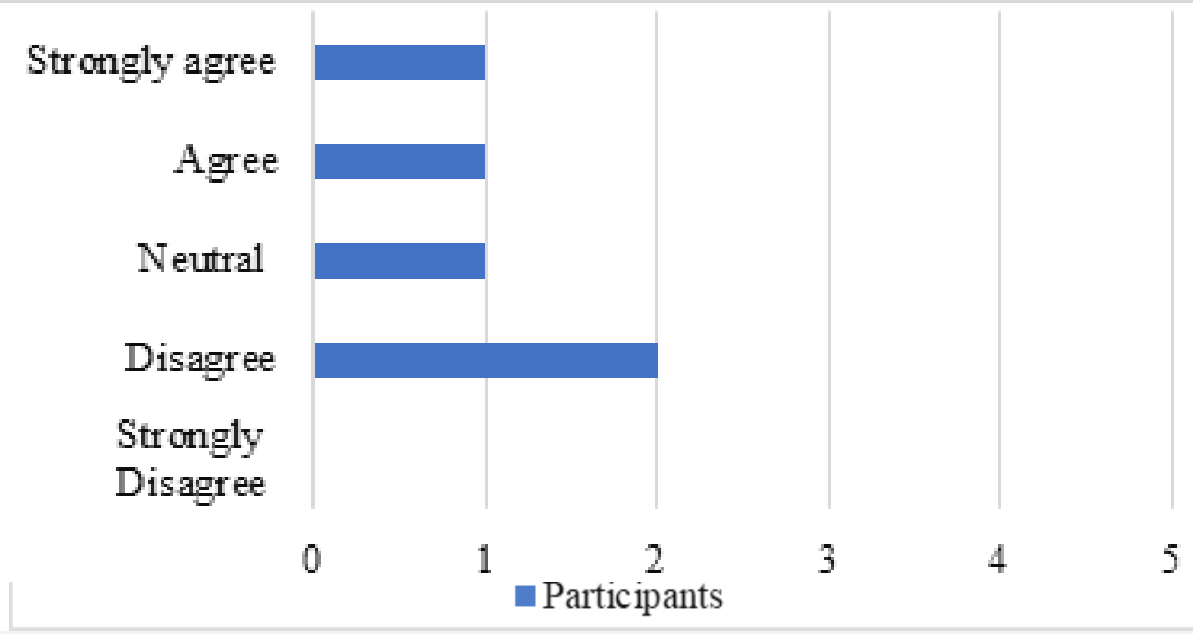

Figure 2: Number of participants indicating "I felt confident in my abilities to care for my loved one after discharge."

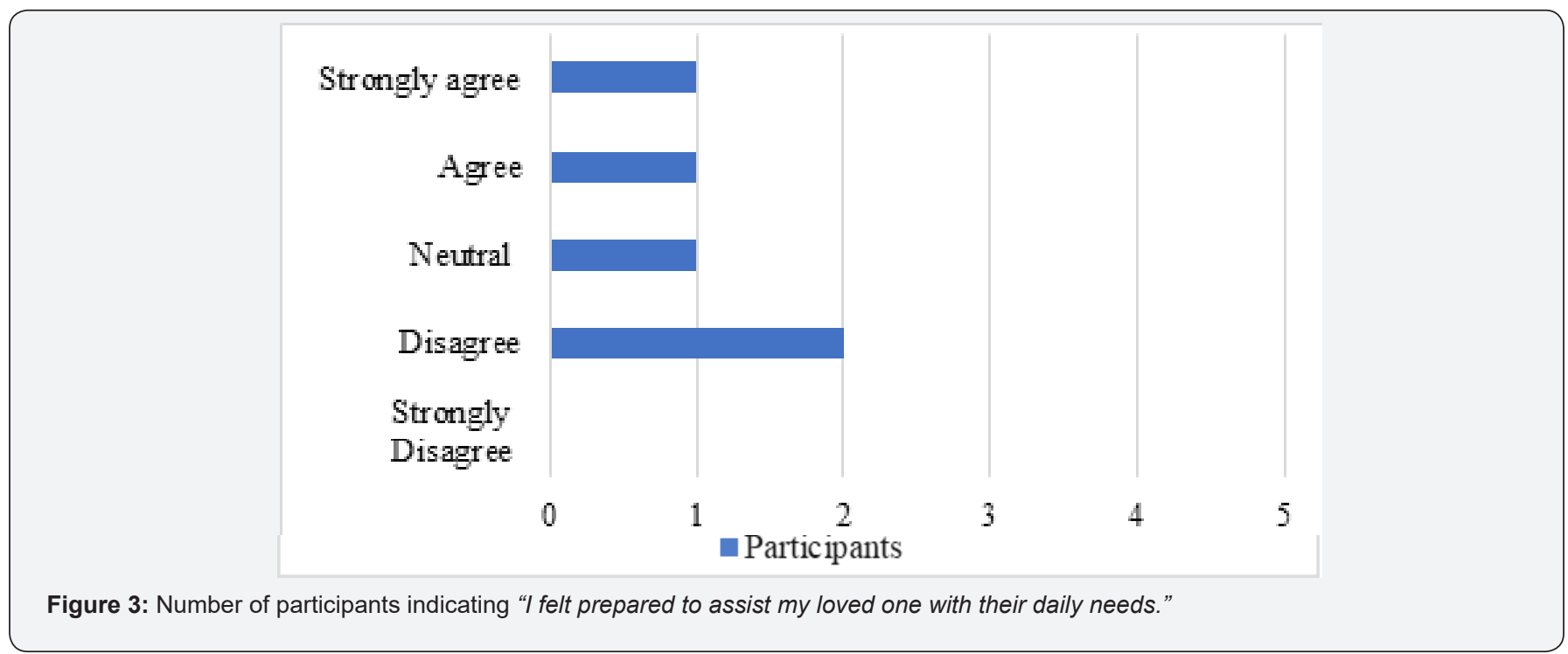




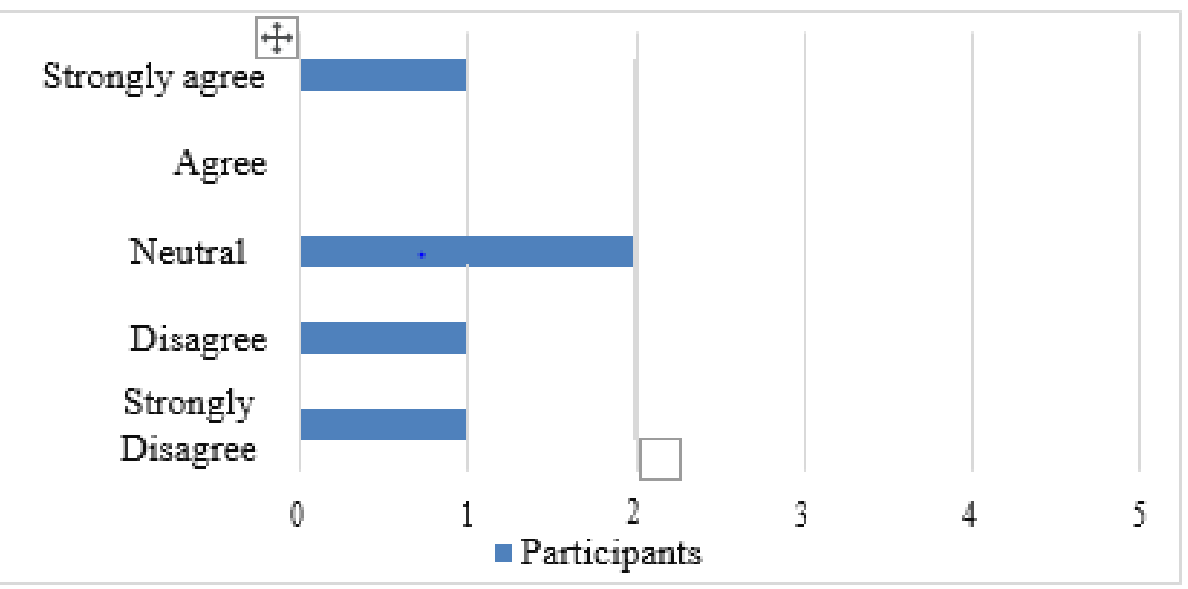

Figure 4: Number of participants indicating "I felt unprepared to assist my loved one with their daily needs."

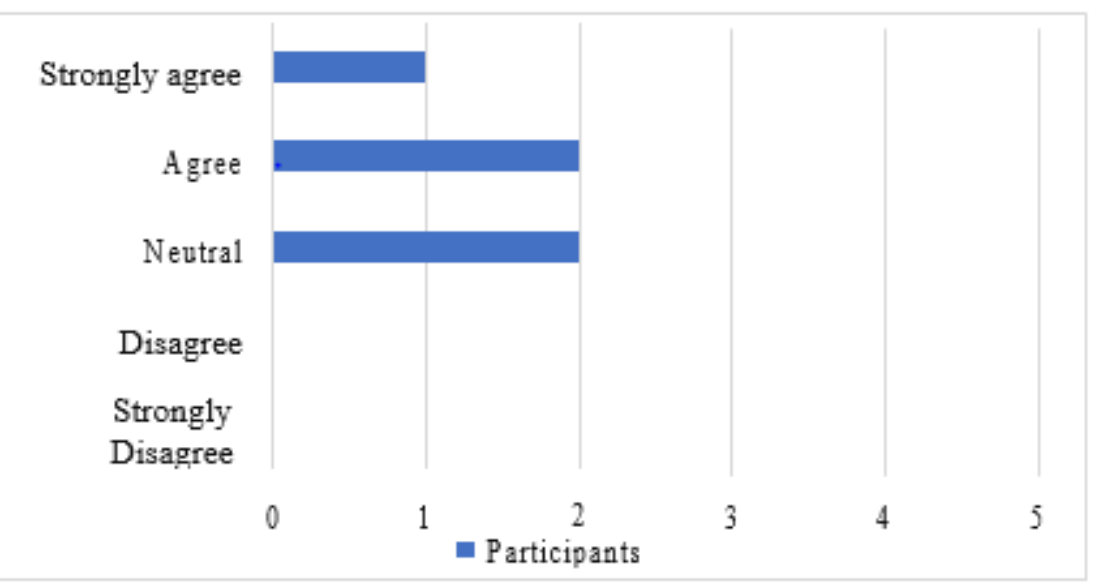

Figure 5: Number of participants indicating "The OT provided education that was tailored to my needs/ learning style."

Table 1: Demographic Data of Participants.

\begin{tabular}{|c|c|c|c|c|c|}
\hline & Gender & Age & Education Level & Current Employment Status & Time Since Stroke \\
\hline Kelly* & F & 80 or older & Graduate degree & Retired & 8 years \\
\hline Samantha* & F & $60-69$ & High school degree or equivalent & Retired & 1.5 years \\
\hline Dorothy* & F & $50-59$ & Graduate degree & Employed, working 40 hours per week & 7 years \\
\hline Kathryn* & F & $70-79$ & Graduate degree & Retired & 3.5 years \\
\hline Abby* & F & $50-59$ & Bachelor's degree & Employed, working 21-39 hours per week & 2 years \& 1 year \\
\hline
\end{tabular}

${ }^{*}$ Pseudonyms were used. 
In terms of the topics addressed by the occupational therapist during the caregiver education session(s) prior to discharge, $100 \%$ of the participants $(n=5)$ indicated that the occupational therapist educated them on bathing and safety; $80 \%(n=4)$ of the participants indicated that that occupational therapist provided education on feeding, grooming, dressing, toileting, and transfers; $60 \%$ of the participants $(n=3)$ reported that they received education on health management and communication management; $40 \%$ of the participants $(n=2)$ stated that they received education on driving; and $20 \%$ of the participants $(n=1)$ specified that they received education on financial management, sleep, meal preparation, and shopping. In terms of the method(s) used to provide the caregiver education, $100 \%$ of the participants $(n=5)$ said it was done through verbal explanation and physical demonstration; $80 \%(n=4)$ indicated that they had an opportunity to practice the newly learned skill; $20 \%$ of the participants $(n=1)$ stated that they received written information during their caregiver education session(s); and $0 \%$ of the participants $(n=0)$ received videotaped resources as part of their caregiver education. In regard to the amount of OT caregiver educational sessions, $40 \%$ of the participants $(n=2)$ indicated that they received their OT caregiver education during the week of discharge, with one of those two participants receiving only one session of OT caregiver education during the week of discharge; $20 \%$ of the participants $(n=1)$ reported that they received OT caregiver education at the week of admission and at the week of discharge; and $40 \%$ of the participants $(n=2)$ specified that they received OT caregiver education throughout the rehabilitation stay. Additionally, 100\% of the participants $(n=5)$ revealed that they received OT caregiver education after discharge from their OT in home health $(n=1)$, day rehabilitation $(n=2)$, or out-patient care $(n=2)$.

\section{Qualitative data}

Three themes emerged from the qualitative data: (1) strengths of education provision; (2) shortfalls; and (3) future recommendations for practitioners.

\section{Strengths of education provision}

Caregivers identified two methods employed by OTs as key in fostering comfort in their abilities after discharge. These included therapists' establishment of a strong rapport with the caregiver in addition to their intentional emphasis towards providing a clientcentered education.

Many caregivers felt the existence of a strong rapport played a central part in fostering a sense of authority with their new role. The participants described their OT's as being genuine, open, caring, and thoughtful. This relationship was illustrated through a variety of unique, yet similar experiences. Such examples included OTs listening to the caregiver's needs, opinions, and concerns; welcoming families to attend sessions; or simply providing genuine reassurance as therapy progressed. For instance, Samantha stated:
"We loved all the occupational therapists...we went to all of her therapy sessions with her Monday through Friday for ten weeks, so we got to know all of the therapists, and we watched everything that they did, so that when we went home, you know, we knew what she needed to do, and you know, where she was and what level... and at [facility's name] they do encourage family to attend therapy sessions...I feel their person ableness was key."

Whereas, Abby, in response to what the caregiver appreciated the most about the education provided by the OT, she stated:

"I think just trying to make you just as comfortable as possible about this experience, knowing you'll be able to do this, even though you're going to hit challenges. Ensuring that everything will be fine. It's just overwhelming and scary that this is a new way of life and you just don't know what to expect when you get home."

Moreover, caregivers also identified therapists' efforts to provide an education that addressed their ever-changing needs and concerns as valuable. Participants described how these client-centered interactions helped induce a renewed sense of competency and comfort in their performance of certain duties. For example, Dorothy expressed:

"The weekend before he was discharged, we had like a trial visit. So we went through everything that we would need to do on a daily basis, and if we had any questions or issues, we brought them to the people that were helping, and they helped us figure out ways to get through any problems we had...so we felt comfortable when we went home."

Whereas Abby explained that:

"The showering was the biggest issue for us...The OT he had there was just amazing. He was so impressive, and he had all these new ideas on what we could do with the shower situation...I took pictures...he (the OT) said 'No, you need to add more bars here, this would be safer for him with the bars here.' Then he said, 'You know, he could just wrap a towel around his neck,' because you know, I always have to get him a towel when he is done showering and get everything for him. And (the OT) was like 'Why? Just tell him to put the towel around his neck and hang it over the top of the shower curtain and then just pull it down when he's done."

\section{Shortfalls of education provision}

A few methods employed by OTs were seen as futile in promoting comfort and confidence by caregivers. Participants described how OTs often demonstrated and ran through caretaking strategies only once prior to discharge. This left caregivers anxious and overwhelmed about certain tasks related to their new role. For example, Kathryn stated: "I didn't know what to do with him...I had to figure out how to get him in the shower, and they pretty much only showed me only once how to do it". Similarly, Samantha, announced that "at the time she was incontinent, and you know I did practice that (toileting) at the hospital, but only like once or twice, and when I got home, of course when you are 
on your own...that is what was difficult for me." Likewise, Kathryn explained that even though she was present for many of the OT sessions, she did not feel like she got enough practice and training when she reported that "... was visiting and it is was time for OT... you know I would observe and everything, but there was never a one on one with just the OT asking, you know what questions I had, or what I should be sure that he does at home."

Additionally, all five of the participants reported how they never received any information about psychosocial resources from the OT, although some found themselves in significant need of them. These resources were especially sought-after when changes in mood, emotions, or behavior began to impact their loved one's daily occupational functioning. For example, Abby described: "I actually called his doctor about (psychosocial resources) because I didn't know what to do, because he did go into a depression, and I couldn't get him to do anything. I couldn't get him to shower or get out of bed. I didn't know what to do." Whereas Samantha indicated that she never received any information regarding support groups or resources in the community. She said: "I think (the facility) has an outreach program, but we never looked into that because it was an hour away...it would have been nice to be connected to some resources within my own community..." Likewise, Kathryn proclaimed that "we had to find that ourselves."

Other caregivers expressed how generic written material possessed little value in the days after discharge. For example, Kelly reported:

"She gave us some written materials too. I have to be honest; I haven't really looked at that... just don't have time to go through all of that. It is all very overwhelming as it is. I would write down some notes to write down on my own if I needed it."

\section{Future recommendations for practitioners}

Participants suggested several methods in which they believe would help promote greater feelings of preparedness in caregivers after discharge. First, all participants expressed the need for more opportunities to practice caretaking strategies taught by OTs prior to discharge. For example, Abby stated: "Like I needed a couple of walkthroughs, and he needed a couple more walkthroughs, to figure out how to do this [showering] safely." Other participants expressed a desire for more opportunities to problem-solve with OTs, whether it is following a trial run at home prior to discharge or through other means several days or weeks out from rehabilitation. For example, Kelly said: "I do like the idea of taking them home at least once to show them the problems we encountered. I think that would have been helpful." and Kathryn proclaimed that follow up after discharge would be beneficial when she said "Follow up with a phone call at home. Just asking how things are going. Maybe provide another educational session where we could come back to the hospital and express some of the problems that we are facing at home."
Caregivers also conveyed a desire for more psychosocial resources from OTs. Participants particularly expressed their desire for knowledge regarding social experiences that could introduce them to individuals facing a similar situation. For example, Samantha illustrated the utility of such a connection: "If there was something else, like another caregiver, that was in my situation that we could bounce ideas off of, that would be very helpful!" Whereas Dorothy articulated that online resources (versus handouts on paper) would be extremely beneficial. She declared that "what I would recommend is some online place with online resource links...it could have every scenario that people encounter...information...stroke support group links..."

Moreover, caregivers expressed a want for occupation-based ideas that could help facilitate their loved one's recovery. For example, Kelly described:

"I have a granddaughter who is funny. She came to visit and she played cards with (the stroke survivor), which I would have never thought of" and she said, 'This will help grandpa use his hand.' I would have never thought of that and it was wonderful...Stuff like that! To have a list of things, maybe, that you could do to use the impaired parts. That would be helpful...outside the walking and folding clothes."

\section{Discussion}

The purpose of this study was to explore caregivers' perceptions regarding their comfort and confidence in performing their new role as a caregiver as well as to explore the particular educational methods that caregivers found most beneficial. While the results of this study suggest that OTs employ methods that promote caregivers' comfort and confidence in their abilities postdischarge, these findings also elucidate how these actions need to be further enhanced in order to fully address caregivers' needs.

In opposition to the literature that characterizes interpersonal exchanges between caregivers and providers as discouraging, uncomfortable, and one-sided $[3,9,6]$, participants in this study overwhelmingly described their interactions with OTs as genuine, open, caring, and thoughtful. These traits were found to be important and a significant contributor towards increasing their comfort in their newly assumed caregiver role. Despite research revealing that such positive interactions helped facilitate more individualized care [9], $40 \%$ of the participants in this study indicated that they were neutral about whether the education provided by OTs was tailored to their needs. This may suggest that even though the positive personality traits of the OTs are greatly appreciated and are advantageous in enhancing the caregiver's level of comfort, it may also indicate that the OTs might be assuming that the needs of the patient are the same as the needs of the caregiver, and may be neglecting to address some of the unique and specific needs of the caregiver. Thus, it seems imperative that the OT practitioner take the time to ensure that they are truly 
addressing the needs of both the patient and caregiver. That is, OTs should assess if they are truly collaborating with the caregiver and ensuring that they are providing individualized education that is tailored to the specific needs of each individual caregiver. This, in turn, will help promote a greater sense of comfort and confidence in the caregiver's ability to perform their new role.

Similarly, the results of this study also appear to support the current evidence which suggests providers tend to deliver education that is chiefly driven by its practicality for clinical practice, rather than built upon caregivers' needs and the development of their abilities [11]. This negatively impacts outcomes related to caregivers' comfort in their overall abilities post-discharge. For example, quantitative findings from this study revealed that only $40 \%$ of participants felt confident in their abilities to care for their loved one and only $40 \%$ of the participants felt prepared to assist with their loved one's daily needs after receiving education from an OT. Again, this suggests that caregivers' needs are not being fully addressed and highlights the need for improvement in the caregiver educational process. For example, the qualitative findings revealed that the caregivers often found the provision of information as rather brief, and fervidly recommended that OTs allow more opportunities to practice caretaking strategies prior to discharge. These results echoed current literature in which caregivers described how they felt overwhelmed with the exorbitant amount of information that health professionals tended to provide in the few days prior to discharge $[2,10,11]$. These results suggest that outcomes regarding caregivers' comfort and confidence in their new role will continue to struggle, and likewise fail to improve, if OTs do not make some greater efforts to address the caregivers' educationrelated needs.

More specifically, OTs should ensure that they meeting the caregivers' preferences in relation to delivery, time, and content of education in order to promote better learning and carry over into the home environment. For instance, participants in this study identified that more opportunities to practice newly learned skills, follow-up phone calls and additional educational sessions after discharge, as well as other forms of educational media (e.g., on-line resources and videos) would be advantageous in terms of being able to perform their caregiver role. It is also noteworthy to mention that since all of the participants in this study revealed that their education was performed with verbal explanation and physical demonstration, yet only $20 \%$ reported the receipt of written instructions and none of the participants received videotaped resources or online resources, it seems that it might be beneficial to investigate the use and perceived value of the application of advanced technology in the caregiver training process. Given the ever-changing advances in technology, it seems that these types of materials may be extremely beneficial in reinforcing OT educational efforts and the information learned during caregiver education, yet the application of these materials in OT education appears to be minimal and lagging behind the technological advances.

Moreover, consistent with current evidence, the results of this study suggest that OTs are not adequately providing information prior to discharge that may help caregivers facilitate their loved one's recovery or overcome challenges once they return to the home environment. For example, participants reported how they desire more ideas about beneficial occupation-based and clientrelevantactivities in which they can perform with their loved one to support recovery at home. Additionally, caregivers also expressed a wish for more opportunities to problem-solve with OTs after discharge, especially if a weekend home visit was not feasible for their rehabilitation timeline. This response was complemented by the quantitative finding that revealed how $20 \%$ of participants felt unprepared to address challenges that arose once home with their loved one. Overall, these results paralleled other studies where caregivers discussed how it would be advantageous to continue the educational process beyond discharge, whether through participation in educational sessions on an outpatient basis or in the community $[10,17,12]$. According to caregivers, this would present opportunities for individuals to seek advice regarding new problems or concerns they unexpectedly face as they transition back into the home without the frequent aid of a practitioner $[12,14,19]$.

Furthermore, the results of this study also emphasize the importance of addressing the psychosocial aspects of the caretaking role in education as well. For example, $60 \%$ of participants in this study reported feeling anxious in regard to caretaking abilities, a phenomenon that is commonly discussed in current literature $[8,20]$. Nevertheless, consistent with previous studies, caregivers in this study identified how they were not provided with resources prior to discharge that could aid with the emotional aspects of their assumed role $[6,9,20]$. Altogether, these findings suggest that OTs are not sufficiently providing caregivers with the tools that are crucial towards supporting their role after discharge. If OTs hope to foster more confident and resilient caregivers, they must consider and address caregivers' everchanging needs throughout the continuum of care and beyond the conclusion of services.

\section{Strengths and Limitations}

This study addresses the gap in the literature in regard to what methods employed by OTs are valuable in promoting individuals' confidence and comfort in their new role as caregivers postdischarge. More specifically, the findings of this study provide a better understanding of current methods employed by OTs that are perceived as beneficial and additional methods that would help enhance caregivers' confidence in the future. This research, however, is subject to several limitations. The results of this study may not be transferable due to its small and heterogeneous participant pool. Another limitation could be related to recall bias, as the participants received the education one to eight years prior to this study. Altogether, these limitations restrict the generalizability of these findings to the greater population. 


\section{Future Research Suggestions}

Future studies should include a larger sample size, greater diversity, and an inclusion criterion of a caregiver of someone who had a stroke within the past year. To build upon the findings of this study, future studies should also consider exploring potential means that could aid therapists in providing more individualized education for caregivers in clinical settings (e.g., intake surveys of caregivers' preferences). Researchers may want to further investigate caregivers' preferences in relation to delivery, time, and content of education in order to develop more effective interventions that result in greater confidence in their assumed role.

\section{Conclusion}

This study revealed that OTs interpersonal skills and therapeutic use of self was advantageous in promoting caregivers' confidence and comfort in their caretaking abilities. However, the results of this study also indicate that OTs need to more consistently tailor their OT education to the caregivers' specific and unique needs. OTs need to acknowledge that learning a new skill is challenging and is often not mastered in one day or one session. Thus, it is essential that OTs consider incorporating more opportunities for collaboration with caregivers and for caregivers to be able to practice their new skills numerous times so that they can be involved in the problem-solving process and achieve an acceptable level of comfort and skill. Additionally, the inclusion of psychosocial resources, advanced forms of technology (video tapes and online resources), as well as occupation-based and client-relevant activities to help facilitate their loved one's recovery, may be extremely beneficial for the patient and caregiver. The researchers of this study hope these revelations will not only result in better education for caregivers of stroke survivors, but also aid in providing a greater sense of assurance for individuals during an often unpredictable and unsettling time [21] (Table 2).

Table 2: Participant Responses to Questions Related to Caregiver Education and Self-perception of Caretaking Abilities Post-discharge.

\begin{tabular}{|c|c|c|c|c|}
\hline Questions & \multicolumn{4}{|c|}{$\%$ of Participants } \\
\hline $\begin{array}{l}\text { "The occupational therapist provided education that was tailored to my } \\
\text { needs" }\end{array}$ & $20 \%$ Strongly Agreed & $40 \%$ Agreed & $40 \%$ Neutral & \\
\hline "I felt anxious in regard to my caretaking ability" & 40\% Strongly Agreed & $20 \%$ Agreed & $20 \%$ Neutral & $20 \%$ Disagreed \\
\hline "I felt confident in my abilities to care for my loved one after discharge" & 20\% Strongly Agreed & $20 \%$ Agreed & $20 \%$ Neutral & $40 \%$ Disagreed \\
\hline "I felt prepared to assist with my loved one's daily needs" & $20 \%$ Strongly Agreed & $20 \%$ Agreed & $20 \%$ Neutral & $40 \%$ Disagreed \\
\hline "Once home with my loved one, new problem arose left unprepared for" & $20 \%$ Strongly Agreed & & $40 \%$ Neutral & 40\% Disagreed \\
\hline
\end{tabular}

\section{Appendix A}

\section{Stroke Caregiver Education Provided by Occupational Therapists: Caregivers' Perspective Survey}

Participant Demographic Information:

1. Please indicate your gender.

_. Male

__Female

__other (please specify)

__Prefer not to say

2. Which category below includes your age?

-18-20

-21-29

-30-39

-40-49

50-59

_60-69

- $70-79$ 
_ 80 or older

3. What is the highest level of school you have completed or the highest degree you have received?

__ Less than high school degree

__High school degree or equivalent (e.g., GED)

__Some college but no degree

_. Associate degree

__Bachelor's degree

__Graduate degree

4. Which of the following categories best describes your employment status?

__Employed, working less than 20 hours per week

__Employed, working 21-39 hours per week

__Not employed, looking for work

__Not employed, NOT looking for work

__Retired

If employed, what it your current job title/position? If not employed or retired, write N/A

Questions regarding stroke/ education experience in hospital:

5. How many months/years ago did your loved one experience a stroke?

6. At what hospital/rehab center did you receive caregiver education from an occupational therapist immediately following your loved one's stroke?

7. Please indicate activities that were addressed by the occupational therapist during your education session(s) prior to your loved one's discharge from the hospital. Mark all that apply.

__Feeding

__Grooming/Personal hygiene (e.g. hair, cosmetics, teeth, etc.)

__Dressing

__Tolieting

__Bathing/showering

__Transfers (e.g. wheelchair, bed, car, shower, toilet, chair, etc.)

__Safety (e.g. in the home, out in the community)

__Health management \& maintenance (e.g. nutrition, physical fitness, medication routines)

__Financial management

__Communication management (e.g telephones, computers, tablets)

__Sleep

__Shopping

__Meal preparation

__Driving and community mobility (e.g. public transit, walking) 
8. What methods did the occupational therapist use/provide during these education session(s)?

Mark all that apply.

_-Verbal

_.Physical demonstration

__Opportunity to practice newly learned skills

_-Video

__Brochures/Pamphlets

__other (please specify)

9. How many education session(s) did you have with the occupational therapist prior to your loved one's discharge from the hospital?

_one

_ Two

__Three

__other (please specify)

10. When did these education sessions occur?

_The week of discharge

__At admission and discharge

__Throughout the rehabilitation stay

Questions regarding experience post-discharge:

11. Did you receive caregiver education in another care setting by an occupational therapist after your loved one's discharge from the hospital? Mark all that apply.

__Home-health

__Day-rehab

__outpatient therapy

__other (please specify)

For the following questions, please fill in the bubble that best represents your opinion of the statement given:

12. In the hospital, the occupational therapist adequately provided education that was tailored to my needs/learning style:

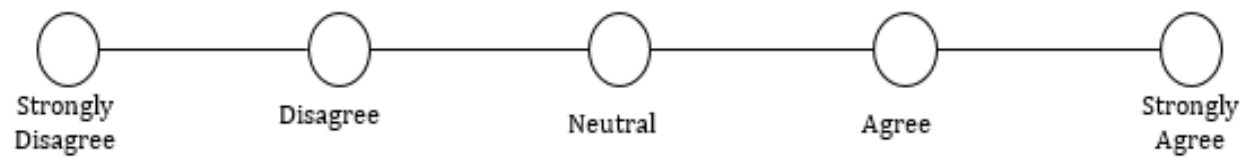

13. Following my loved one's discharge from the hospital, I felt confident in my abilities to care for his or her needs in the home environment:

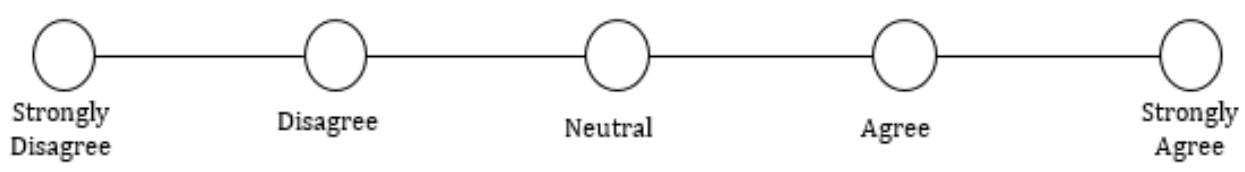


14. Following my loved one's discharge from the hospital, I felt anxious in regard to my caretaking ability:

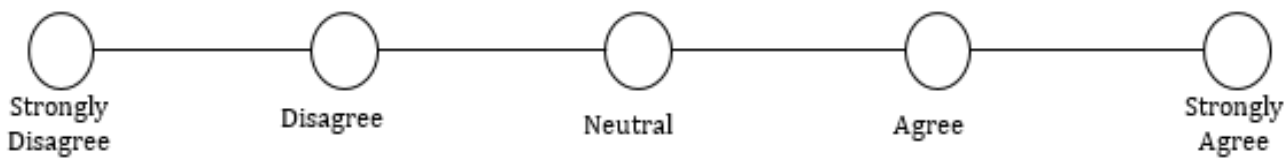

15. Following my loved one's discharge from the hospital, I felt prepared to assist with their daily needs:

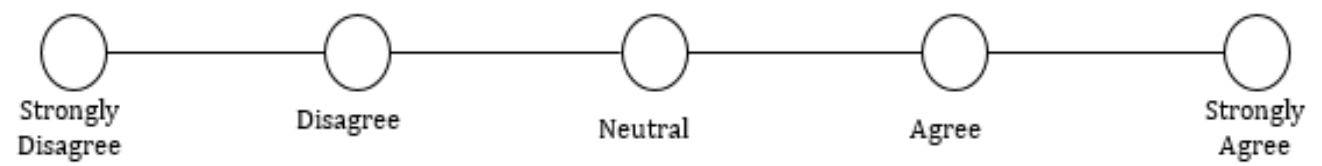

16. Once in the home environment with my loved one, new problems arose that I felt unprepared to handle and desired further education on:

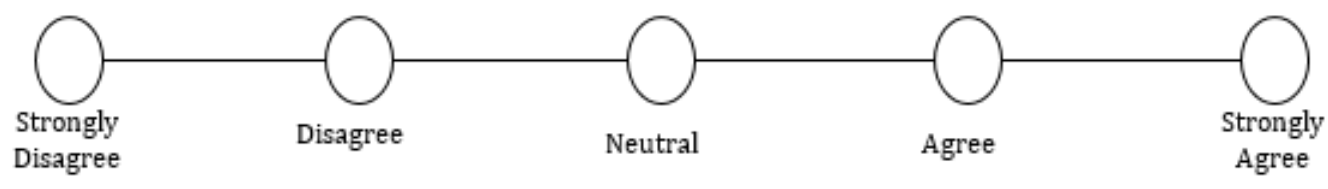

\section{Appendix B}

\section{Focus Group Interview Guide}

Stroke Caregiver Education: From the Caregivers' Perspective

1. Did you feel confident in your abilities to care for your loved one when returning home after discharge?

Probe: Did you feel anxious about performing certain activities in the home environment without the aid of the therapist?

2. What was your experience with the collaboration that occurred between you and the occupational therapist during the stroke education process prior to discharge?

Probes: Did the therapist seek out and/or listen to your opinions/needs/concerns during the education process prior to discharge? Did the therapist ask whether the teaching methods being used were effective to your learning?

3. Before being discharged, were you informed about the possible changes in behavior, moods, and emotions of your loved one and how to manage these changes?

Probes: Were you provided with resources in the community such as other social support groups?

Were you surprised or did you feel unprepared to address some of the changes in mood/emotion/behavior after discharge?

4. Did occupational therapists join you at your home before discharge in order to practice the transition? Was this effective?

Probes: At the hospital, did the occupational therapist demonstrate and practice methods to help your loved one with transfers before discharge. (e.g. bed, toilet, shower chair, etc.)

At the hospital, did the occupational therapist demonstrate and practice methods to help your loved one with ADLs before discharge? (e.g. dressing, eating, grooming, bathing, toileting )

Were you able to go home for a few days before being discharged from the hospital to practice some of the things you learned in therapy at home? Did this help you feel more comfortable/ skilled/ confident in your role as a caregiver?

5. Which education strategies utilized by the Occupational Therapist did you find most helpful?

Probes: How did the occupational therapist provide information to you? Written

materials? Verbal instruction? Video? Demonstration? Were you given time to practice after the therapist demonstrated a technique?

If so, did you find that material helpful, or did it help change the focus of caregiver education? 


\section{What changes do you want to see for delivery of caregiver education in the future?}

Probe: What teaching methods do you feel would have increased your sense of preparedness to perform caretaking duties?

\section{References}

1. Hankey G (2013) Training caregivers of disabled patients after stroke. The Lancet 382: 2043-2044.

2. Ghazzawi A, Kuziemsky C, O Sullivan T (2016) Using a complex adaptive system lens to understand family caregiving experiences navigating the stroke rehabilitation system. BMC Health Services Research 16: 1-10.

3. Shook R, Stanton S (2016) Patients' and caregivers' self-perceived stroke education needs in inpatient rehabilitation. International Journal of Therapy \& Rehabilitation 23(6): 278-287.

4. Camicia M, Lutz BJ, Markoff N, Catlin A (2018) Determining the needs of family caregivers of stroke patients during inpatient rehabilitation using interview, art, and survey. Rehabilitation Nursing: The Official Journal of the Association of Rehabilitation Nurses 44(6): 328-337.

5. Cheng HY, Chair SY, Chau JPC (2018) Effectiveness of a strengthoriented psychoeducation on caregiving competence, problemsolving abilities, psychosocial outcomes and physical health among family caregivers of stroke survivors: A randomised controlled trial. International Journal of Nursing Studies 87: 84-93.

6. Roy D (2015) Health professional and family perceptions of poststroke information. Nursing Praxis in New Zealand 31(2): 7-24.

7. Chen L, Xiao LD, De Bellis A (2016) First-time stroke survivors and caregivers' perceptions of being engaged in rehabilitation. Journal of Advanced Nursing 72(1): 73-84.

8. Creasy KR, Lutz BJ, Young ME, Ford A, Martz C (2013) The impact of interactions with providers on stroke caregivers' needs. Rehabilitation Nursing: The Official Journal of the Association of Rehabilitation Nurses 38(2): 88-98.

9. Danzl MM, Harrison A, Hunter EG, Kuperstein J, Sylvia V, et al. (2016) "A lot of things passed me by:" Rural stroke survivors' and caregivers' experience of receiving education from health care providers. The J Rural Health 32(1): 13-24.

10. Banford M, Kratz M, Brown R, Emick K, Ranck J, et al. (2001) Stroke survivor caregiver education: Methods and effectiveness. Physical \& Occupational Therapy in Geriatrics 19(1): 37-51.
11. Eames, S, Hoffmann T, Worrall L, Read S (2010) Stroke patients' and carers' perception of barriers to accessing stroke information. Topics in Stroke Rehabilitation 17(2): 69-78.

12. Cameron JI, Gignac MAM (2008) “Timing it right:" A conceptual framework for addressing the support needs of family caregivers to stroke survivors from the hospital to the home. Patient Education and Counseling 70(3): 305-314.

13. Allison R, Evans HP, Kilbride C, Campbell LJ (2008) Secondary prevention of stroke: Using the experiences of patients and carers to inform the development of an educational resource. Family Practice 25(5): 355 .

14. Luker J, Murray C, Lynch E, Bernhardsson S, Shannon M, et al. (2017) Carers' experiences, needs, and preferences during inpatient stroke rehabilitation: A systematic review of qualitative studies. Archives of Physical Medicine \& Rehabilitation 98(9): 1852e13-1862e13.

15. Wachters-Kaufmann C, Schuling J, The H, Meyboom-de-Jong B (2005) Actual and desired information provision after a stroke. Patient Education \& Counseling 56(2): 211-217.

16. O'Connell B, Baker L, Prosser A (2003) The educational needs of caregivers of stroke survivors in acute and community settings. Journal of Neuroscience Nursing 35(1): 21-28.

17. Creswell JW, Plano Clark VL (2011) Designing and conducting mixed methods research (2nd edn) Sage.

18. Braun V, Clarke V (2006) Using thematic analysis in psychology. Qualitative Research in Psychology 3(2): 77-101.

19. Ing MM, Linton KF, Vento MA, Nakagawa K (2015) Investigation of stroke needs (INVISION) study: Stroke awareness and education. Hawaii J Med Public Health 74(4): 141-145.

20. Knecht-Sabres L (2016) Caregivers of stroke survivors: Exploring the role of spousal caregivers through an occupational therapy lens. IJAHSP14(3): 4.

21. Sweetman M (2016) Authentic leadership in occupational therapy. OT Practice 21(13): CE-1-CE-8. 
This work is licensed under Creative Commons Attribution 4.0 License DOI: 10.19080/OAJGGM.2021.06.5556868
Your next submission with Juniper Publishers will reach you the below assets

- Quality Editorial service

- Swift Peer Review

- Reprints availability

- E-prints Service

- Manuscript Podcast for convenient understanding

- Global attainment for your research

- Manuscript accessibility in different formats ( Pdf, E-pub, Full Text, Audio)

- Unceasing customer service

Track the below URL for one-step submission https://juniperpublishers.com/online-submission.php 